International Journal on Information Sciences and Computing, Vol. 4, No.2, July 2010

\title{
AN ANALYSIS OF STABILITY OF TRENDS IN MUTUAL FUNDS USING FRACTAL DIMENSION INDEX (FDI) COMPUTED FROM HURST EXPONENTS
}

\author{
Priyadarshini E. ${ }^{1}$ and Chandra Babu A. ${ }^{2}$ \\ ${ }^{1}$ Research Scholar, Sathyabama University, Chennai-119. \\ ${ }^{2}$ Dept of Mathematics, MNM Jain Engineering College, Chennai-97, \\ E-mail:priyaeb@gmail.com, chandrababu_a@yahoo.co.in
}

\begin{abstract}
The general belief is that the NAV's of the mutual funds take a random and unpredictable path and that it is impossible to outperform the market without assuming additional risk. However, it is possible to outperform the market by carefully selecting entry and exit points for equity investments. Chaos is a nonlinear, dynamic system that appears to be random but is actually a higher form of order. All chaotic systems have a quantifying measurement known as a fractal dimension. The fractal dimension index (FDI) is a tool that applies the principles of chaos theory and fractals. With FDI one can determine the persistence or anti-persistence of any equity or commodity. In this paper we study the data from mutual funds by computing the fractal dimension index. The fractal dimension index is computed from the Hurst exponent, which is computed from Rescaled Range R/S.
\end{abstract}

Keywords: chaos, fractals, rescaled range, persistence.

\section{INTRODUCTION}

A time - series is a set of ordered observations $x_{1}, x_{2}, \ldots x_{i}, x_{i+1}, \ldots x_{n}$ which are equally spaced over time or space. A biased random time - series is characterised by long term dependence or a "memory" between observations. The events of one period influence all the periods that follow. Rescaled Range $(R / S)$ analysis is a technique which is frequently applied to real-time problems to detect any biases in behavior over time. This method is used to find the memory effect, otherwise known as persistence in the trends. Rescaled Range Analysis (R/S Analysis) is a non parametric methodology developed by H.E. Hurst, a British hydrologist in 1951. He applied this methodology to study the long-term storage capacity of reservoirs and later it was extended to study many other natural systems. This statistical methodology is generally used for distinguishing random time series from biased random time series (Fractal time series) and to study the persistence of trends and also the presence of periodic and non-periodic cycles in a time series.

\section{History of Rescaled Range}

British dam builder and hydrologist H.E. Hurst (1900 - 78) worked on the Nile River Dam Project in the early period of 20th century. In an effort to solve a hydrological problem, Hurst searched for patterns in the Nile Delta. The problem involved the storage capacity of the dam reservoir. Most hydrologists assumed that water inflow was a random process with no underlying order. Hurst came to a different conclusion after studying almost a

millennium of Nile overflows. He found that large overflows tend to be followed by larger overflows. There appeared to be cycles, but their lengths were non-periodic and standard statistical analysis revealed no patterns between observations. Hurst developed his own analytical method to explain the non-periodic cycles. To identify a nonrandom process, he tested the Nile using Albert Einstein's work on Brownian motion, a widely accepted model for a random walk. Einstein found that the distance a random particle travels increases with the square root of time used to measure it. This is called the $T^{1 / 2}$ rule, and is commonly used in finance and economics. Hurst divided the Nile data into segments and examined the logarithmic range and scale of each segment in comparison to the total number of segments. This process is called re-scaled range analysis. The range is re-scaled because it has a zero mean and is expressed in terms of local standard deviation.

The rescaled range value scales with an increase in the time increment by a power-law value equal to $\mathrm{H}$, or the Hurst exponent. Using re-scaled range analysis, Hurst showed that water overflows tended to repeat, meaning that the natural overflows were partially predictable. Then Benoit Mandelbrot used the Hurst exponent to experiment with time series found in cotton prices. He developed a method to measure 
irregular natural objects and named the measurement the fractal dimension.

\section{Chaotic systems}

Chaos is present everywhere: in lightning, weather patterns, earthquakes, and financial markets. Though it may appear to be a random event, it is not. Chaos is a nonlinear, dynamic system that appears to be random but is actually a higher form of order. Natural and Social systems, including private, governmental, and financial institutions fall within this category. People design these complex systems only to find that these systems take on a life of their own. Each of the system networks is sustained by complex feedback, loops that re-enter the system at unpredictable points in their cycles. These feedback loops create an illusion of randomness.

In Chaos theory, we try to define an apparently random event in the marketplace that has some degree of predictability. In order to do this we need a tool that is a representation of order from chaos. Fractal is the tool that we will use here. The fractal is commonly defined as an object with self-similar individual parts. In the markets, a fractal can be thought of as an object or "time series" that appears similar across a range of scales. Markets look this way when we compare a 3 minute time scale to a 30 minute time scale and a 30 minute time scale to a 3 day time scale. Each frame may zigzag a little differently, but when viewed from afar they have similar attributes on each scale.

All chaotic systems have a quantifying measurement known as a fractal dimension. The fractal dimension is a non-integer dimension that describes how an object takes up space. Objects in space are infinitely complex. If we examine any object with a microscope, more detail is revealed as the scale changes. In addition to levels of detail, most objects in nature demonstrate self-similarity, the organizing principal of fractals

- Because of this, fractals will maintain their dimension regardless of the scale used. This is evident in natural phenomena such as mountains, coastlines, clouds, hurricanes and lightning. Similarity across scales is essential in trading, because each time frame of a market will have a similar fractal pattern. This also proves that markets are natural phenomena rather than mechanical processes. Such markets can only be forecasted reliably with principles applicable to nonlinear, natural systems using Fractal geometry as tool.

\section{Fractal Dimension Index (FDI)}

The fractal dimension index (FDI) is a tool that applies the principles of chaos theory and fractals. This specialized indicator identifies the fractal dimension of the market by using re-scaled range analysis and an estimated Hurst exponent. It does so by using all available data on the time/price chart to determine the "volatility" or "trends" of a given market. FDI is the same type of tool used by eminent fractal scholars Benoit Mandelbrot, H.E. Hurst, and Edgar Peters in their examinations of time series analysis. With FDI we can determine the persistence or anti-persistence of any equity or commodity that we display in our graphing program. A persistent time series will result in a chart that is less jagged, subject to fewer reversals, and resembles a straight line. An anti-persistent time series will result in a chart that is more jagged and prone to more reversals. Essentially, FDI will tell us whether a market is a random, independent system or one with bias.

The FDI is useful because it determines the amount of market volatility. The easiest way to use this indicator is to understand that a value of 1.5 suggests that the market is acting in a completely random fashion. As the market deviates from 1.5, the opportunity for earning profits increases in proportion to the amount of deviation. The entire scale is based on a range of $1-2$, suggesting extreme linearity to extreme volatility. A great example that is often used to describe the fractal dimension is its use in geography. If we examine an island and plot the fractal dimension, we will be able to determine how jagged the edges of the island are for a particular measurement scale. An island with a 1.7 fractal dimension is highly jagged, with many more peaks and troughs on the periphery. An island with a fractal dimension of 1.3 is much more linear, approaching a single dimension or a straight line. If we examine this island on a map, the coastline will be straighter.

Because the price plot on chart will never be one extreme or another, we need to measure the "fraction of the dimension." This is why the FDI number is a fractal dimension. The farther away this dimension is from 1.5, the more confident one can be that the market is not random. When a market is not random, 
it is more predictable. If the FDI is closer 2, the probability is higher that the next move will be in the opposite direction of the current move. An FDI closer to 1 signals a trending market in one direction.

This knowledge alone gives the trader an incredible advantage, because it can indicate which markets have the most opportunity. While trading a basket of Forex markets, futures or stocks we certainly do not want to include those markets near a 1.5 FDI. If none are close to 1.5 we can still determine the market(s) with the largest degree of "predictability" by omitting those closer to 1.5 .

Another postulate is the length of time necessary for each data period. Some researchers believe that shorter time periods, such as daily data, are subject to more noise from random information. If this is the case, FDI is less accurate with finer slices of sequential data. The individual length of each period will come into play as noise is filtered.

\section{Computing the FDI}

For computing the FDI one must first compute the Hurst exponent. For a time series $x_{1}, 2,,, x_{n}$ of length $n$, Hurst computed the Rescaled Range $\left(R / S_{n}\right)$ and found that

$$
(R / S)_{n}=c^{*} n^{H}
$$

where $c$ and $H$ are constants and $H$ is the Hurst coefficient.

$$
\log \left(\left(R / S_{n}\right)=\log (c)+H^{*} \log (n)\right.
$$

Thus $H$ is obtained as the slope of the Log $\left(R / S_{n}\right)$ versus $\log (n)$ plot. $H=0.50$ implies that the time series is a random time series and implies the absence of long term statistical dependence. The case $0.50<H<=1.00$ implies a persistent time series, a time series characterized by long memory effects. This means that if the trend has been positive in the last observed period, the chances are that it will continue to be positive in the next period. Conversely if it has been negative in the last period it is more likely that it will continue to be negative in the next period. The level of persistence is judged by how far $H$ is above 0.5. Hurst has found that a number of natural phenomenon such as river discharges, rainfall, sunspot numbers and tree rings exhibit a Hurst coefficient $H$ with mean 0.73 and a standard deviation 0.09 , showing that past values influence the present values. The case $0<=H<0.50$ implies anti-persistent time series. An anti persistent system reverses itself more frequently than a random one.

The length $n$ is now increased to the next higher value, such that $(N-1) / n$ is an integer and then $(R / S)_{n}$ is computed. The pairs of values log $(n)$ and are plotted in a graph sheet and the line of best fit (given by equation (2)) is drawn. The slope $H$ of this line is the estimate of the Hurst coefficient.

After the computation of the Hurst exponent, we derive the fractal dimension of the time series. This is easily accomplished using the formula $D=2-\mathrm{H}$. "2"is used here because we are using two dimensions for this computation. The value of " $D$ " is the fraction of a dimension between 1 and 2 that the price data represents. Logarithmic returns are used to compute FDI. Because logarithmic returns sum to cumulative returns, most analyst agree that this is most appropriate for financial analysis.

\section{Correlation between periods:}

The correlation $C_{N}$ between periods is calculated as follows:

$$
C_{N}=2^{(2 H-1)}-1
$$

A random time series $(H=0.5)$ has zero correlation. A persistent time series (with $H$ greater than 0.5 ) results in positive correlation where as an anti-persistent time series results in negative correlation. $C_{N}$ is a measure of the long-term memory present in the time series. $C_{N}=0.25$ implies that $25 \%$ of the data is influenced by the past.

\section{The V Statistic:}

The $V$ statistic

$$
V_{n}=\frac{(R / S)_{n}}{\sqrt{n}}
$$

was originally used by Hurst for testing stability. If the process is an independent random process, then the plot of $\mathrm{V}$ versus Log $\mathrm{n}$ will be flat. If the process is persistent $(H>0.5)$ then the graph will be upwardly sloping and if the process is anti persistent $(H<0.5)$ the graph will be downward sloping. 


\section{Data Analysis}

The data for analysis is taken from six of the top ten mutual funds for the time period Jan 2008 to Dec 2008.

1. Birla Sunlife Asset Allocation Aggressive

2. FT India life Stage FOF 20's

3. Sahara Growth

4. UTI Contra

5. Escorts High Yield Equity

6. HDFC Top 200

Birla Sunlife Asset Allocation Aggressive
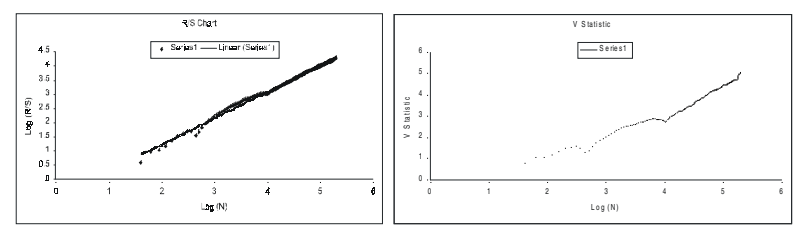

$$
H=0.9196 \quad C_{H}=0.789 \quad F D=1.0804
$$

FT India life Stage FOF 20's

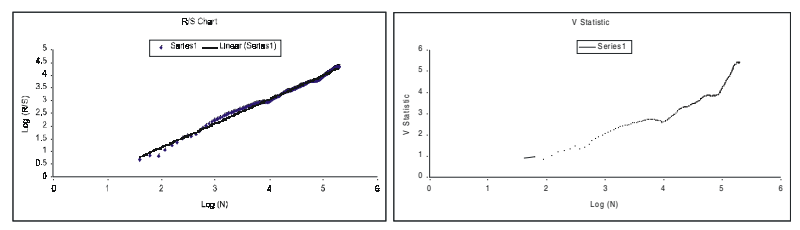

$$
H=0.9431 \quad C_{H}=0.8484 \quad F D=1.057
$$

Sahara Growth

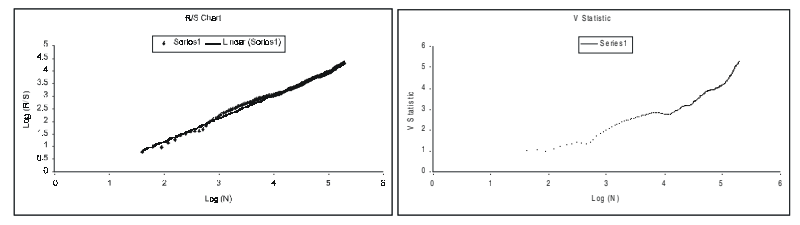

$$
H=0.9203 \quad C_{H}=0.7908 \quad F D=1.078
$$

UTI Contra
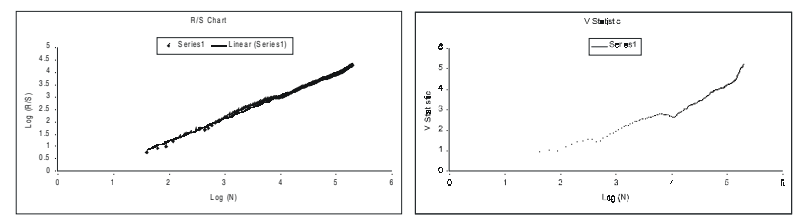

$H=0.911 \quad C_{H}=0.7679 \quad F D=1.089$

\section{Escorts High Yield Equity}

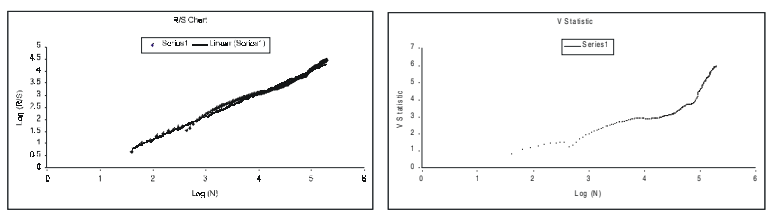

$H=0.948 \quad C_{H}=0.862 \quad F D=1.052$

HDFC Top 200

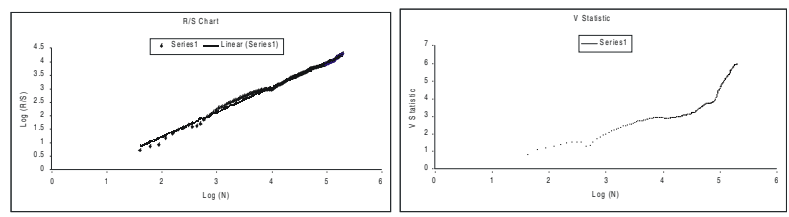

$$
H=0.913 \quad C_{H}=0.774 \quad F D=1.087
$$

9. Final Result

\begin{tabular}{|c|l|c|c|c|}
\hline No & $\begin{array}{l}\text { Name of } \\
\text { Mutual } \\
\text { Fund }\end{array}$ & $\begin{array}{c}\text { Hurst } \\
\text { constant }\end{array}$ & $\begin{array}{c}\text { Correlation } \\
\text { between } \\
\text { period }\end{array}$ & $\begin{array}{c}\text { Fractal } \\
\text { Dimension } \\
\text { Index }\end{array}$ \\
\hline 1 & $\begin{array}{l}\text { Birla } \\
\text { Sunlife } \\
\text { Asset } \\
\text { Allocation } \\
\text { Aggressive }\end{array}$ & 0.919 & 0.789 & 1.0803 \\
\hline 2 & $\begin{array}{l}\text { FT India } \\
\text { Life } \\
\text { Stage } \\
\text { FOF 20's }\end{array}$ & 0.943 & 0.848 & 1.057 \\
\hline 3 & $\begin{array}{l}\text { Sahara } \\
\text { Growth }\end{array}$ & 0.920 & 0.791 & 1.078 \\
\hline 4 & $\begin{array}{l}\text { UTI } \\
\text { Contra }\end{array}$ & 0.911 & 0.768 & 1.089 \\
\hline 5 & $\begin{array}{l}\text { Escorts } \\
\text { High } \\
\text { Yield } \\
\text { Equity }\end{array}$ & 0.948 & 0.862 & 1.052 \\
\hline 6 & $\begin{array}{l}\text { HDFC } \\
\text { Top 200 }\end{array}$ & 0.913 & 0.774 & 1.087 \\
\hline
\end{tabular}

10. Conclusion

From the study carried out on the six of the top ten mutual funds, it was found out that All these mutual 
funds were highly persistent, which resulted in a chart that is less jagged, prone to fewer reversal, resembling a straight line and clearly fractals in nature. The plot of $V_{n}$ versus $\log n$ for these funds has upward slope confirming its persistence and the correlation between periods are positive indicating the presence of long term memory. Since the FDI for all these mutual funds are closer to 1, they are not random and the trending market is predictable in one direction. It was also observed that the mutual fund Escorts High Yield Equity has the highest $H$ value while UTI Contra has the lowest $H$ value. Therefore we can conclude that EHYE is less risky than UTI Contra.

\section{BIBLIOGRAPHY}

[1] Edgar.E.Peters, 1994 "Fractal Market Analysis", John Wiley and Sons, New York, pp 189-196.

[2] Edgar.E.Peters, 1991 "Chaos and Order in Capital Markets", John Wiley and Sons, New York.
[3] Edgar.E.Peters, 1989 "Fractal Structure in the Capital Markets", Financial Analysts Journal (July -Aug 1989) pp32-37.

[4] Mandelbrot.B, 1982 "The Fractal Geometry of Nature", New York, W.H.Freeman.

[5] Mandelbrot.B, 1972 "Statistical Methodology for non-periodic cycles from the covariance's of $\mathrm{R} / \mathrm{S}$ Analysis", Annals of Economics and Social measurement.

[6] Barnsley.M, 1988 "Fractals everywhere", Boston M A etc: Academic Press, Inc.

[7] Davies,R.B. and Harte, D.S. "Test for Hurst effect", Biometrica, 74: 95-102.

Mrs. E. Priyadarshini, M.Sc. M.Phil. P.G.D.C.A. is a senior lecturer (mathematics) in Sathyabama University. She has completed 18 years of teaching experience in various colleges. At present she is doing her research at Sathyabama University. 